\title{
New evidence for halo gas accretion onto disk galaxies
}

\author{
Filippo Fraternali ${ }^{1}$ \\ ${ }^{1}$ Astronomy Department, University of Bologna, via Ranzani 1, I-40127, Bologna, Italy \\ email: filippo.fraternali@unibo.it
}

\begin{abstract}
Studies of the halo gas in the Milky Way and in nearby spiral galaxies show the presence of gas complexes that cannot be reconciled with an internal (galactic fountain) origin and are direct evidence of gas accretion. Estimating gas accretion rates from these features consistently gives values, which are one order of magnitude lower than what is needed to feed the star formation. I show that this problem can be overcome if most of the accretion is in fact "hidden" as it mixes with the galactic fountain material coming from the disk. This model not only provides an explanation for the missing gas accretion but also reproduces the peculiar kinematics of the halo gas in particular the vertical rotation gradient. In this view this gradient becomes indirect evidence for gas accretion.
\end{abstract}

\section{Introduction}

A large amount of fresh gas accretion onto the Milky Way has been advocated for decades since the work of Twarog (1980) showed the relative constancy of the Star Formation Rate (SFR) in the Galactic disk over the last 10 Gyrs. Roughly constant SFR requires constant accretion and this translates today into a need for gas accretion at a rate of the order $\sim 1 M_{\odot} \mathrm{yr}^{-1}$.

Since the 1960s, the presence of a large number of neutral hydrogen (H I) clouds filling the sky around us and having, on average, negative velocities with respect to the Milky Way disk has been recognized (e.g. Hulsbosch 1968). Such clouds, named High Velocity Clouds (HVCs), given their largely anomalous radial velocities (Wakker \& van Woerden 1997), were immediately regarded as possible evidence for gas accretion from intergalactic space onto our Galaxy (Oort 1970). Alternative interpretations have been proposed, for example that the clouds are produced by the cooling of material expelled from the disk via a "galactic fountain" (Bregman 1980) or that they are a much more distant Local Group population (e.g. Blitz 1999).

From the time of Oort's suggestion until very recently there were two major unknowns about the HVCs: their distances and their metallicities. It is thanks to a tenacious observational campaign carried out especially over the last few years that we now know both distances and metallicities for all the major HVCs (Wakker et al. 2007, 2008). The results leave no doubts: the HVCs are located in the Milky Way halo and have metallicities of about an order of magnitude lower than the average disk ISM metallicity. These properties make it most likely that this is accreting material falling onto the Milky Way for the first time.

The HVCs do not comprise the entire halo population, rather they are an extreme population at large heights and having particularly anomalous velocities. In the lower halo large amounts of cold gas are observed (e.g. Lockman 1984), some of which go under the name of Intermediate Velocity Clouds (IVCs) and are regarded as a likely galactic fountain component (e.g. Wakker 2001). More recently, Kalberla \& Dedes (2008) showed that up to $10 \%$ of the Milky Way H I gas is in fact extra-planar and highly turbulent. 


\section{Halo gas in nearby spiral galaxies}

In the last decade, the study of the halo (extra-planar) gas has been extended to nearby galaxies. This is a demanding task, given the low surface brightness of the halo emission, and the studies have been restricted to a relatively small number of objects. In Table 1 , I summarize the results for the best studied galaxies so far. This table includes galaxies seen at different inclination angles. For edge-on galaxies the halo gas can be separated spatially from the disk gas, whilst for galaxies seen at intermediate inclinations it can be separated thanks to its peculiar kinematics. The main kinematic feature of extra-planar gas is its decreasing rotation velocity with increasing height from the plane, it is said to be "lagging" behind the disk gas. Such a velocity gradient has been estimated for a few galaxies (column 9, Table 1) and it is an important constraint for models of extra-planar gas formation (see Section 4). The presence of this gradient is also the main reason why extra-planar gas can be detected in non edge-on galaxies (Fraternali et al. 2002).

Table 1. Physical properties of extra-planar gas in spiral galaxies

\begin{tabular}{lccccccccc}
\hline Galaxy & Type & $\begin{array}{c}\text { incl } \\
\left({ }^{\circ}\right)\end{array}$ & $\begin{array}{c}\mathrm{v} f l a t^{(\mathrm{km} / \mathrm{s})} \\
\text { Milky Way }\end{array}$ & $\begin{array}{c}\mathrm{M}_{\mathrm{HI}} \text { halo } \\
\left(10^{8} M_{\odot}\right)\end{array}$ & $\begin{array}{c}\mathrm{M}_{\mathrm{HI}} \text { tot } \\
\left(10^{9} M_{\odot}\right)\end{array}$ & $\begin{array}{c}\mathrm{SFR} \\
\left(M_{\odot} / \mathrm{yr}\right)\end{array}$ & $\begin{array}{c}\text { Accr. rate } \\
\left(M_{\odot} / \mathrm{yr}\right)\end{array}$ & $\begin{array}{c}\text { Gradient }^{a} \\
(\mathrm{~km} / \mathrm{s} / \mathrm{kpc})\end{array}$ & Ref. \\
M 31 & $\mathrm{Sb}$ & 77 & 226 & $\sim 4$ & 4 & $1-3$ & $\approx 0.2^{b}$ & -22 & $(1,2,3)$ \\
NGC 253 & $\mathrm{Sc}$ & $\sim 75$ & $\sim 185$ & 0.8 & 3 & 0.35 & - & - & $(4,5)$ \\
M 33 & $\mathrm{Scd}$ & 55 & 110 & $>0.1$ & 1 & 0.5 & $0.05^{c}$ & - & $(6)$ \\
NGC 2403 & $\mathrm{Scd}$ & 63 & 130 & 3 & 3.2 & 1.3 & 0.1 & $\sim-12$ & $(9)$ \\
NGC 2613 & $\mathrm{Sb}$ & $\sim 80$ & $\sim 300$ & $4.4^{d}$ & 8.7 & 5.1 & - & - & $(10)$ \\
NGC 3044 & $\mathrm{Sc}$ & 84 & 150 & 4 & 3 & $2.6^{e}$ & - & - & $(11)$ \\
NGC 4559 & $\mathrm{Scd}$ & 67 & 120 & 5.9 & 6.7 & $0.6^{e}$ & - & $\sim-10$ & $(12)$ \\
NGC 5746 & $\mathrm{Sb}$ & 86 & 310 & $\sim 1$ & 9.4 & 1.2 & $0.2^{f}$ & - & $(13,14)$ \\
NGC 5775 & $\mathrm{Sb}$ & 86 & 200 & - & 9.1 & $7.7^{e}$ & - & $-8^{g}$ & $(15)$ \\
NGC 6946 & $\mathrm{Scd}$ & 38 & 175 & $\gtrsim 2.9$ & 6.7 & 2.2 & - & - & $(17)$ \\
NGC 891 & $\mathrm{Sb}$ & 90 & 230 & 12 & 4.1 & 3.8 & 0.2 & -15 & $(18)$ \\
UGC 7321 & $\mathrm{Sd}$ & 88 & 110 & $\gtrsim 0.1$ & 1.1 & $\sim 0.01^{h}$ & - & $\gtrsim-25$ & $(19)$ \\
\hline
\end{tabular}

${ }^{a}$ Gradient in rotation velocity with height (from the flat part of the rotation curve); ${ }^{b}$ from complex $\mathrm{C}$ and other clouds with known distances in (2) without correction for the ionised fraction; ${ }^{c}$ from the H I mass in (8) without their correction for the ionised fraction; ${ }^{d}$ from sum of the various extra-planar clouds; ${ }^{e}$ calculated from the FIR luminosity using the formula in Kewley et al. (2002); ${ }^{f}$ from the counter-rotating cloud (13) using an infall time-scale of $1 \times 10^{8}$ yr; ${ }^{g}$ calculated using optical lines $(16) ;{ }^{h}$ SFR of only massive stars $>5 M_{\odot}$. References: (1) Kalberla \& Dedes (2008); (2) Wakker et al. (2007), Wakker et al. (2008); (3) Levine, Heiles \& Blitz (2008); (4) Thilker et al. (2004); (5) Walterbos \& Braun (1994); (6) Boomsma et al. (2005); (7) Reakes \& Newton (1978); (8) Grossi et al. (2008); (9) Fraternali et al. (2002); (10) Chaves \& Irwin (2001); (11) Lee \& Irwin (1997); (12) Barbieri et al. (2005); (13) Rand \& Benjamin (2008) (14) Pedersen et al. (2006); (15) Irwin (1994); (16) Heald et al. (2006a); (17) Boomsma et al. (2008); (18) Oosterloo, Fraternali, \& Sancisi (2007); (19) Matthews \& Wood (2003).

Fig. 1 shows the two types of extra-planar gas detections. On the left, the total H I map of the edge-on galaxy NGC 891 (blue + contours) is overlaid onto a DSS optical image (orange). The H I data (obtained with the Westerbork Synthesis Radio Telescope) show a massive and extended $\mathrm{H}$ I halo with a mass of $1.2 \times 10^{9} M_{\odot}$ or $30 \%$ of the total H I mass (Oosterloo, Fraternali \& Sancisi 2007). On the right panel of Fig. 1, the position-velocity (p-v) plot along the major axis of the galaxy NGC 2403 (inclination $60^{\circ}$ ) obtained with the VLA (Fraternali et al. 2001). A broad component of gas at rotation velocities lower 

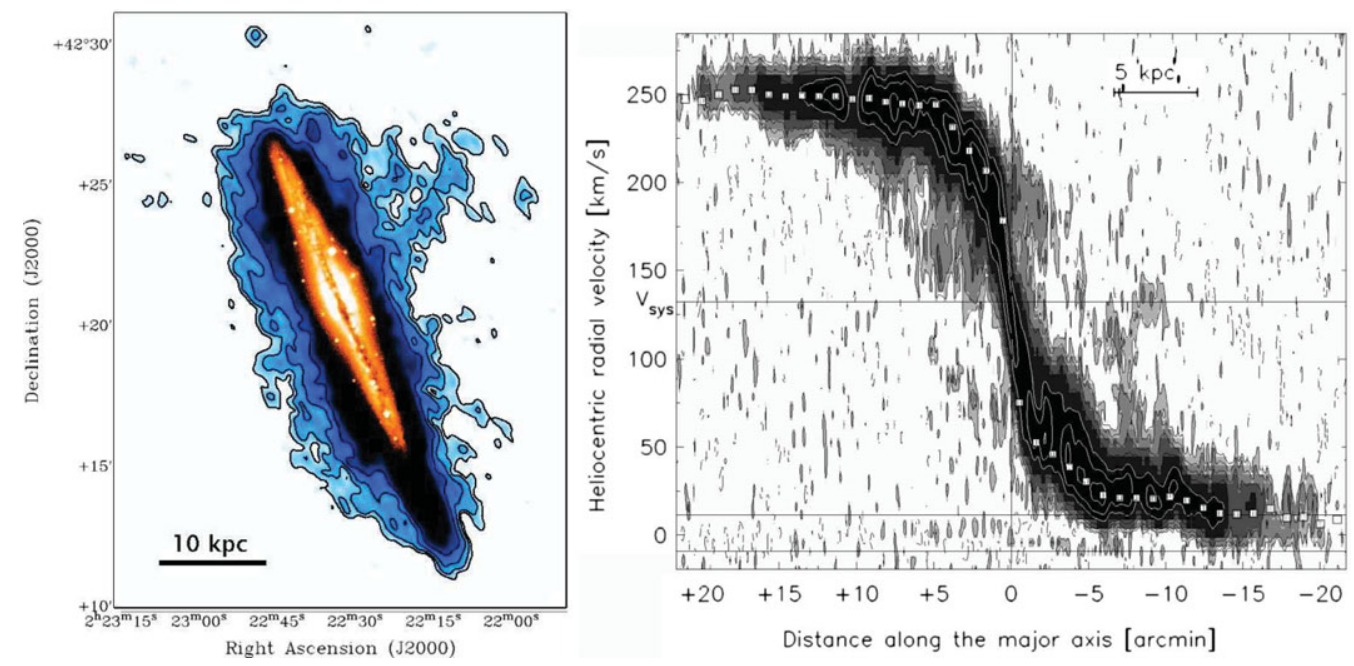

Figure 1. Two methods of detecting extra-planar gas. Left: total H I map (blue + contours) for the edge-on galaxy NGC 891, overlaid on the optical image (orange) (data from Oosterloo et al. 2007). The extra-planar gas is clearly separated on the sky and surrounds the whole galactic disk with a filament that extends up to $20 \mathrm{kpc}$. Right: position-velocity diagram along the major axis of the intermediate inclination galaxy NGC 2403 (from Fraternali et al. 2001). The extra-planar gas is kinematically separated from the disk gas. It is seen as a faint component rotating more slowly than the disk (white dots: = disk rotation curve).

than the disk gas (also called the "beard") is clearly visible at low emission levels (light grey). This beard component is the halo gas in NGC 2403.

Extra-planar gas is possibly ubiquitous as several nearby galaxies other than those reported in Table 1 show indications of its presence. It is also observed in the ionised phase. Optical studies of nearby edge-on galaxies show that roughly half of them have extended layers of diffuse ionised gas (e.g. Rossa \& Dettmar 2003) and with similar kinematics as the H I layers (Heald et al. 2006b).

\section{Direct evidence for gas accretion}

Direct evidence of gas accretion is difficult to obtain. The strategy is to look for gas components (usually at very anomalous velocities) which are incompatible with an internal origin. The large majority of the extra-planar gas studied so far has actually a very regular kinematics that follows closely the kinematics of the disk (see for instance the p-v diagram for NGC 2403, right panel of Fig. 1). This points to a tight connection between disk and halo components.

The first features that deserve attention in the search for gas accretion are the HI filaments. Among the galaxies in Table 1 at least half show large filamentary H I structures in their halos, the most notable cases being NGC 891 and NGC 2403. NGC 891 has a long massive filament $\left(M_{\mathrm{HI}} \sim 1.6 \times 10^{7} M_{\odot}\right)$ extending up to about $20 \mathrm{kpc}$ from the plane of the disk (Fig. 1). NGC 2403 also has a filament with a similar H I mass located in projection outside the bright optical disk and clearly separated in velocity from the disk kinematics (Fig. 2). They are both very similar to Complex C in our Galaxy. We can calculate the energy needed to form these filaments assuming that they come from the disk through a galactic fountain. In the case of the NGC 891 filament it turns out that this energy should be of the order $\sim 1 \times 10^{55} \mathrm{erg}$. This would correspond to the explosion 

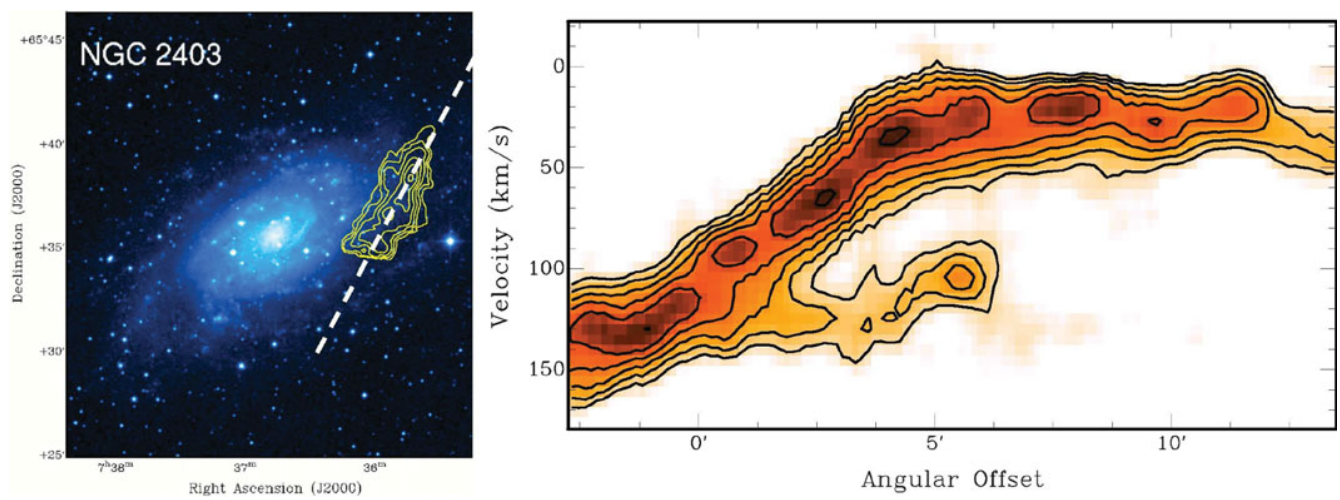

Figure 2. The H I filament in NGC 2403. Left: optical image overlaid with the H I map (in contours) of the $1 \times 10^{7} M_{\odot}$ filament. Right: position-velocity plot along the dashed line in the left panel. The filament is $8 \mathrm{kpc}$ long and separated from the normal disk kinematics (higher contours) by about $90 \mathrm{~km} \mathrm{~s}^{-1}$. Note the small dispersion in velocity of the filament and its proximity to the systemic velocity of the galaxy $\left(v_{\mathrm{sys}}=133 \mathrm{~km} \mathrm{~s}^{-1}\right)$.

of about $10^{5}$ supernovae in a specific region of space and over a time-scale shorter than a dynamical time, which is clearly a very unlikely event.

A second type of feature that has been found in these new deep surveys are clouds at very anomalous velocities that end up in the region of counter-rotation. The data of NGC 891 show two such clouds with masses of order $\sim 10^{6} M_{\odot}$ and counter-rotating velocities of $\sim 50$ and $\sim 90 \mathrm{~km} \mathrm{~s}^{-1}$ (Fig. 3). These clouds cannot be produced in any kind of galactic fountain and they are most likely direct evidence of gas accretion. NGC 2403 also has gas components at very anomalous velocities called "forbidden gas" (Fig. 1; Fraternali et al. 2002). Counter-rotating or forbidden clouds are also observed in NGC 4559, in NGC 5746 and NGC 6946.
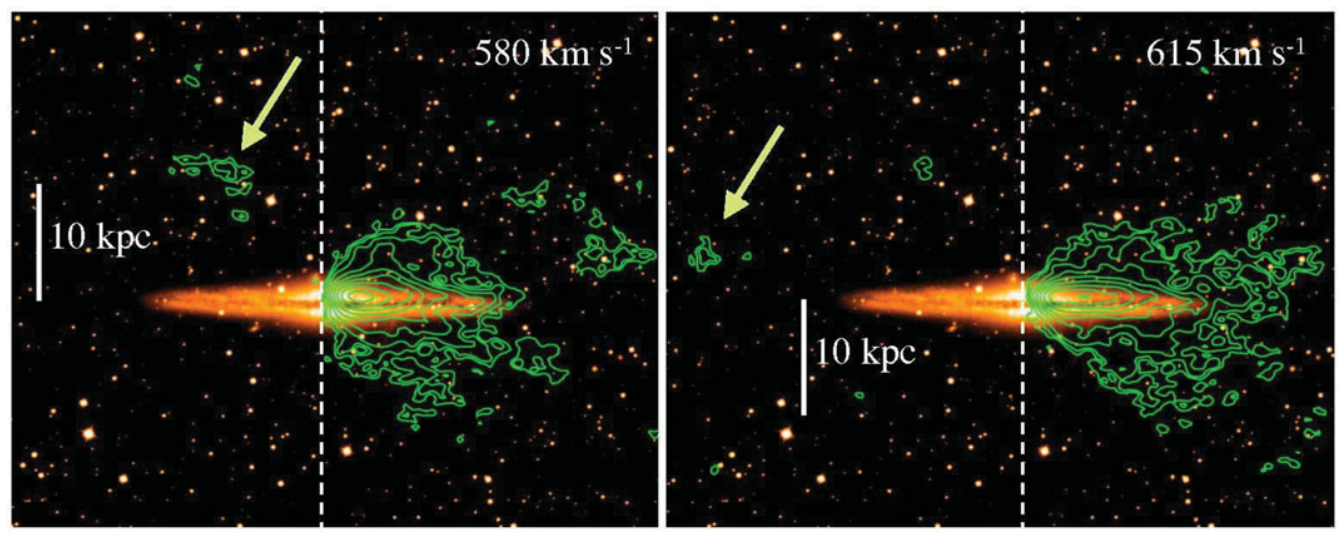

Figure 3. Counter-rotating clouds in NGC 891. These two channel maps at radial velocities 580 and $615 \mathrm{~km} \mathrm{~s}^{-1}\left(v_{\mathrm{sys}}=528 \mathrm{~km} \mathrm{~s}^{-1}\right)$ show gas on the receding side (to the right of the vertical dashed line) of the galaxy. Two clouds are visible in isolation on the left side of the galaxy (arrows), they are counter-rotating (from Oosterloo et al. 2007.

If we believe that the above structures have an extragalactic origin we can estimate the rate of gas accretion by assuming typical infalling times of few $\times 10^{7}-10^{8} \mathrm{yr}$. The resulting rates are shown in Table 1 (column 8 ), they are typically of the order $0.1 M_{\odot} \mathrm{yr}^{-1}$ and generally 1 order of magnitude lower than the SFRs. These directly observed accretion 
rates include only $\mathrm{HI}$, and they should be corrected for helium and possibly ionised gas fractions. However, it appears difficult to reconcile them with the rates of star formation (column 7, Table 1). This result is very common for nearby galaxies (Sancisi et al. 2008).

\section{Indirect evidence for gas accretion}

The result that the rate of gas accretion onto galaxies which is directly observed is much lower than expected implies that most of the accretion should be somewhat "hidden". I describe here possible indirect evidence of this missing gas accretion, provided by the rotation velocity gradient of the extra-planar gas. The steepness of this gradient is not reproduced by galactic fountain models (e.g. Fraternali \& Binney 2006; Heald et al. $2006 \mathrm{~b}$ ) as they tend to predict shallower values (a factor half or less). Fig. 4 highlights this problem for NGC 891. The points are rotation velocities derived at heights $z=3.9 \mathrm{kpc}$ and $z=5.2 \mathrm{kpc}$ from the plane (Fraternali et al. 2005). Clearly the fountain clouds in the model rotate too fast (have a larger angular momentum) than the extra-planar gas in the data.
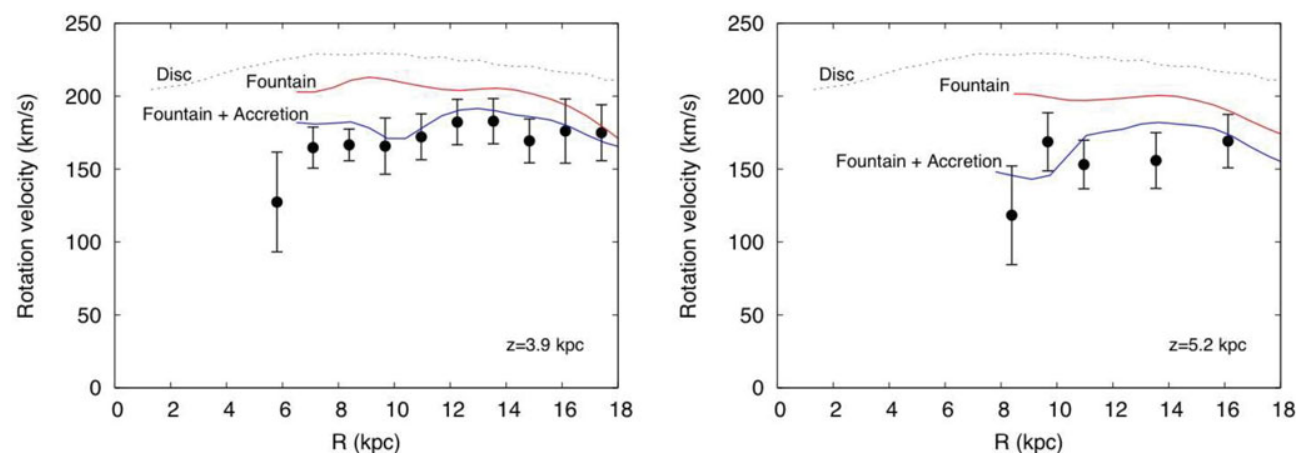

Figure 4. Indirect evidence for gas accretion. Rotation velocities (black points) of the H I extra-planar gas in NGC 891 at $3.9 \mathrm{kpc}$ (left) and $5.2 \mathrm{kpc}$ (right) from the plane compared to the disk rotation curve (dotted line) and predictions from two models: a pure galactic fountain model (red line above) and a model where the fountain clouds sweep up and accrete ambient gas during their passage through the halo (blue line below). The accretion rate required to produce this fit is $\sim 3 M_{\odot} \mathrm{yr}^{-1}$, very similar to the star formation rate of NGC 891 (from Fraternali \& Binney 2008).

How can the fountain clouds loose part of their angular momentum? Fraternali \& Binney (2008) consider the possibility that fountain clouds sweep up ambient gas as they travel through the halo. In this scheme ambient gas condenses onto the fountain clouds, these latter grow along their path through the halo and eventually fall down into the disk (see Fig. 5). If the ambient gas has relatively low angular momentum about the $\mathrm{z}$-axis then this condensation produces a reduction in the angular momentum of the fountain gas. The only free parameter of the model is the accretion rate, which is tuned to reproduce the rotation curves of the extra-planar gas. Remarkably, the required gas accretion rate turns out to be very similar to the SFR. For NGC 891 we found a bestfit accretion rate of about $3 M_{\odot} \mathrm{yr}^{-1}$ (see the blue curves in Fig. 4) and for NGC 2403: $0.8 M_{\odot} \mathrm{yr}^{-1}$. In NGC 2403, this model is also able to reproduce the observed radial inflow of the halo gas (see Fraternali \& Binney 2008).

One implication of the above fountain+accretion model is that it predicts that most of the extra-planar gas is produced by the galactic fountain and only a small fraction (about 10\%) is extragalactic. This is in agreement with the metallicity of the IVCs and 


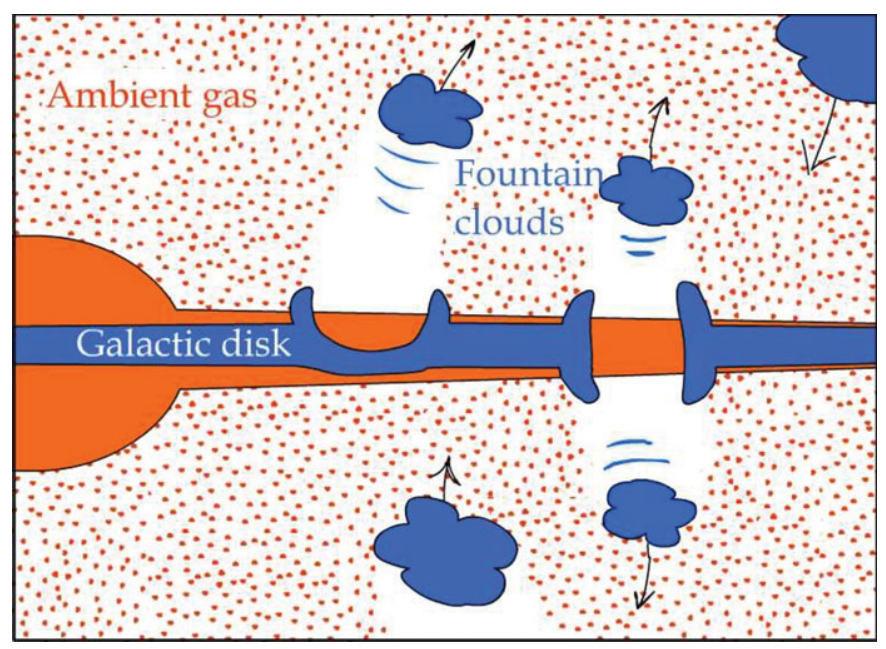

Figure 5. Schematic of the fountain gas sweeping up ambient gas. The model presented in Fraternali \& Binney (2008) suggests that superbubbles blow gas into the halo which then sweeps up part of the ambient gas during its passage before falling back to the disk. This mechanism allows fountain clouds to loose part of their angular momentum as required by the data (see Fig. 4) and produces a net gas accretion at a rate similar to the SFR.

the clear links between anomalous velocity clouds and star forming regions (e.g. Boomsma et al. 2008). A second implication is that it does not require that the accreting gas is in any particular phase but only that its angular momentum about the z-axis is less than about half the angular momentum of the disk material. Finally, this model predicts an accretion rate of the order of the SFR and in general, proportional to the supernova rate. Interestingly, this appears to be a general requirement for galaxies throughout the Hubble time as it reconciles the observed cosmic star formation history with the gas mass in galaxies at low and high redshifts (Hopkins, McClure-Griffiths \& Gaensler 2008).

\section{References}

Barbieri, C. V., Fraternali, F., Oosterloo, T., Bertin, G., Boomsma, R., \& Sancisi, R. 2005, A\&A, 439, 947

Blitz, L., Spergel, D. N., Teuben, P. J., Hartmann, D., \& Burton, W. B. 1999, ApJ, 514, 818

Boomsma, R., Oosterloo, T. A., Fraternali, F., van der Hulst, J. M., \& Sancisi, R. 2005, A\&A, 431,65

Boomsma, R., Oosterloo, T. A., Fraternali, F., van der Hulst, J. M., \& Sancisi, R. 2008, A\&A, in press

Bregman, J. N. 1980, ApJ, 236, 577

Chaves, T. A. \& Irwin, J. A. 2001, ApJ, 557, 646

Fraternali, F., Oosterloo, T., Sancisi, R., \& van Moorsel, G. 2001, ApJ, 562, L47

Fraternali, F., van Moorsel, G., Sancisi, R., \& Oosterloo, T. 2002, AJ, 123, 3124

Fraternali, F., Oosterloo, T., Sancisi, R., \& Swaters, R. 2005, in: ed. R. Braun, Extra-planar Gas, Dwingeloo, ASP Conf. Series, Vol. 331, p. 239

Fraternali, F. \& Binney, J. J. 2006, MNRAS, 366, 449

Fraternali, F. \& Binney, J. J. 2008, MNRAS, 386, 935

Kalberla, P. M. W., Dedes, L. 2008, A\&A, in press (arXiv0804.4831)

Kewley, L. J., Geller, M. J., Jansen, R. A., \& Dopita, M. A. 2002, AJ, 124, 3135

Grossi, M., Giovanardi, C., Corbelli, E., Giovanelli, R., Haynes, M. P., Martin, A. M., Saintonge, A., \& Dowell, J. D. 2008, A\&A, in press (arXiv0806.0412) 
Heald, G. H., Rand, R. J., Benjamin, R. A., Collins, J. A., \& Bland-Hawthorn, J. 2006a, ApJ, 636,181

Heald, G. H., Rand, R. J., Benjamin, R. A., \& Bershady, M. A. 2006b, ApJ, 647, 1018

Hopkins, A. M., McClure-Griffiths, N. M., \& Gaensler, B. M. 2008, ApJ, 682, L13

Hulsbosch, A. N. M. 1968, BAN, 20, 33

Irwin, J. A. 1994, ApJ, 429, 618

Levine, E. S., Heiles, C., \& Blitz, L. 2008, ApJ, 679, 1288

Lee, S.-W. \& Irwin, J. A. 1997, ApJ, 490, 247

Lockman, F. J. 1984, ApJ, 283, 90

Matthews, L. D. \& Wood, K. 2003, ApJ, 593, 721

Oosterloo, T., Fraternali, F., \& Sancisi, R. 2007, AJ, 134, 1019

Oort, J. H. 1970, A\&A, 7, 381

Pedersen, K., Rasmussen, J., Sommer-Larsen, J., Toft, S., Benson, A. J., \& Bower, R. G. 2006, NewA, 11, 465

Rand, R. J., \& Benjamin, R. A. 2008, ApJ, 676, 991

Reakes, M. L., \& Newton, K. 1978, MNRAS, 185, 277

Rossa, J., \& Dettmar, R.-J. 2003, A\&A, 406, 505

Sancisi, R., Fraternali, F., Oosterloo, T., \& van der Hulst, T. 2008, A\&ARv, 15, 189

Thilker, D. A., Braun, R., Walterbos, R. A. M., Corbelli, E., Lockman, F. J., Murphy, E., \& Maddalena, R. 2004, ApJ, 601, L39

Twarog, B. A. 1980, ApJ, 242, 242

Wakker, B. P \& van Woerden, H. 1997, ARA\&A, 35, 217

Wakker, B. P. 2001, ApJS, 136, 463

Wakker, B. P., York, D. G., Howk, C., Barentine, J. C., Wilhelm, R., Peletier, R. F., van Woerden, H., Beers, T. C., Ivezic, Z., Richter, P., \& Schwarz, U. J. 2007, ApJ, 670, L113

Wakker, B. P., York, D. G., Wilhelm, R., Barentine, J. C., Richter, P., Beers, T. C., Ivezić, Z., \& Howk, J. C. 2008, ApJ, 672, 298

Walterbos, R. A. M., Braun, R. 1994, ApJ, 431, 156

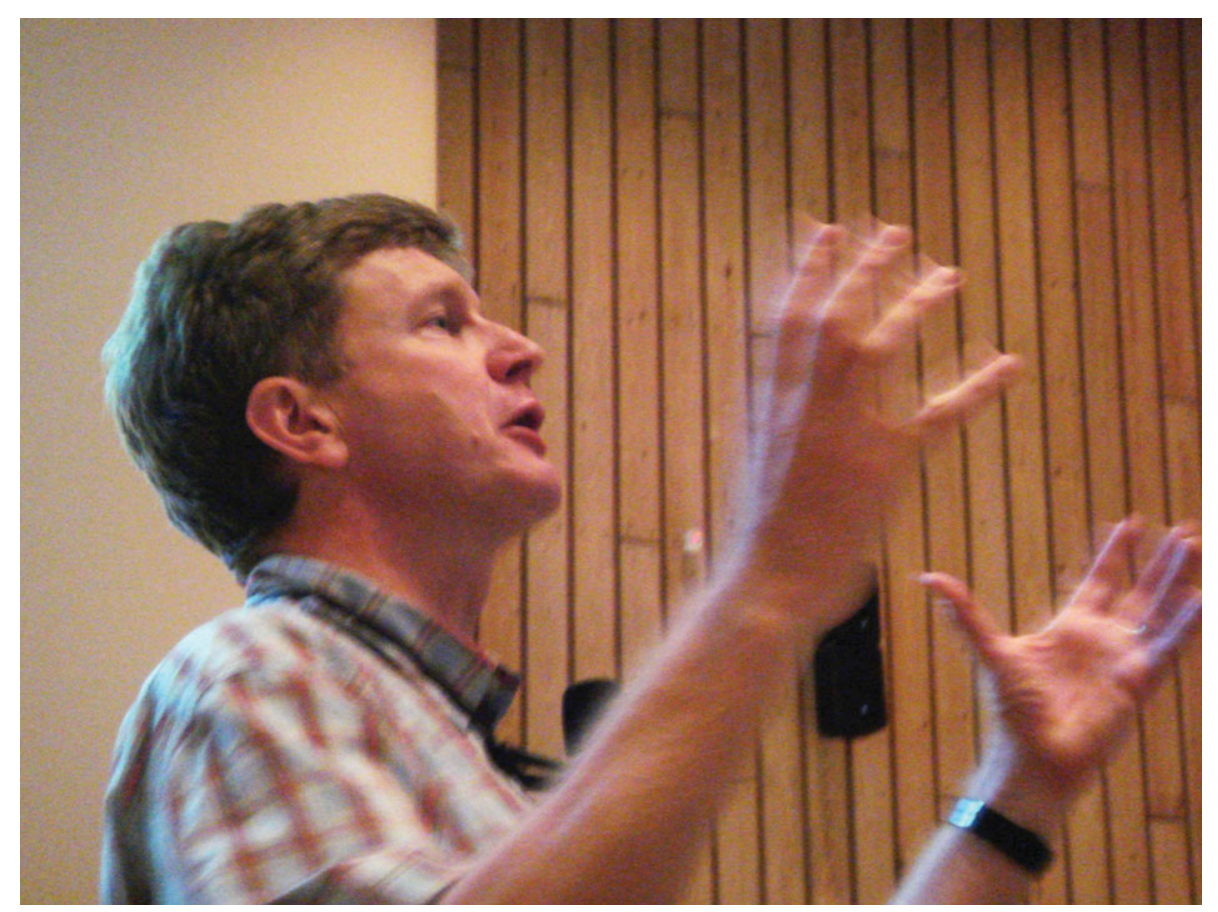

Joss Bland-Hawthorn urging halo gas clouds to fall onto the disk. 


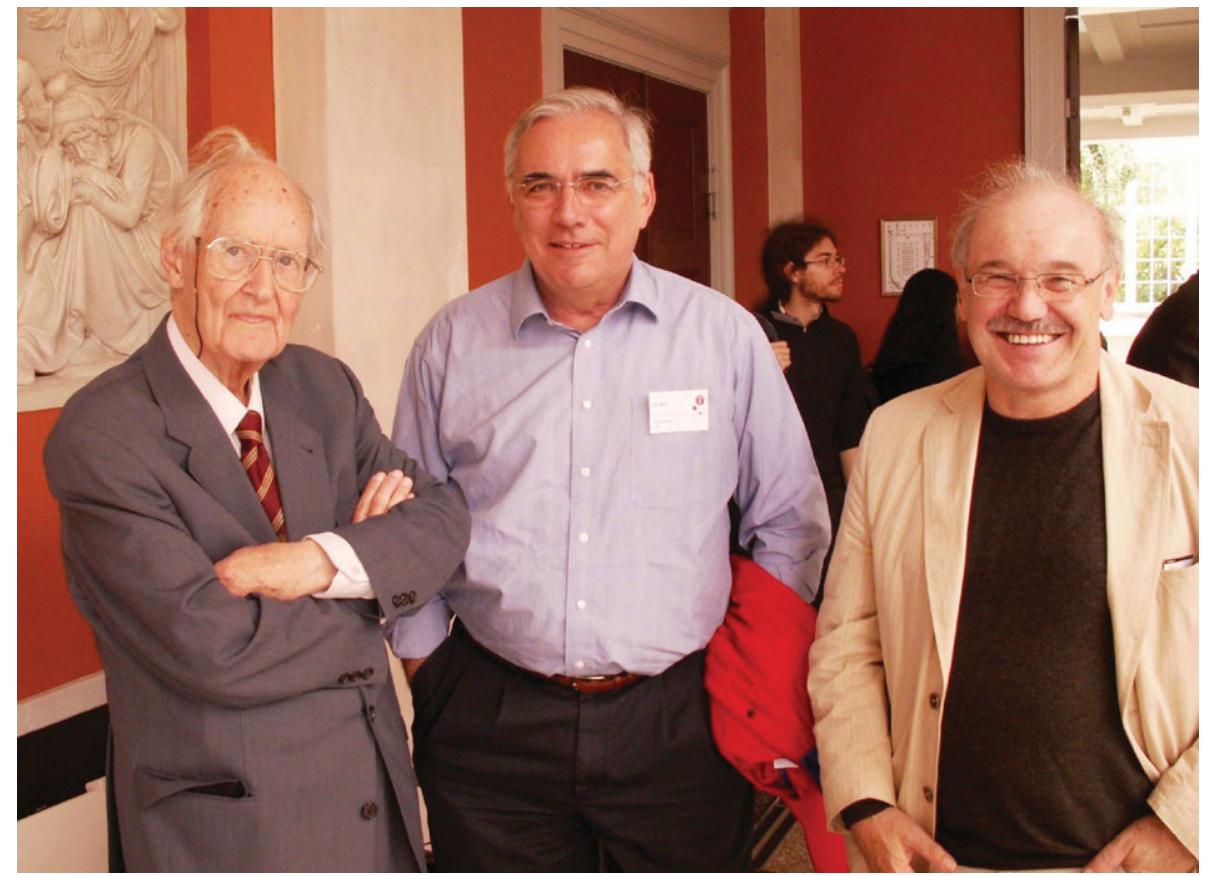

Adriaan Blaauw, Leo Blitz and Hans Zinnecker at Carlsberg (photo: Bruce Elmegreen).

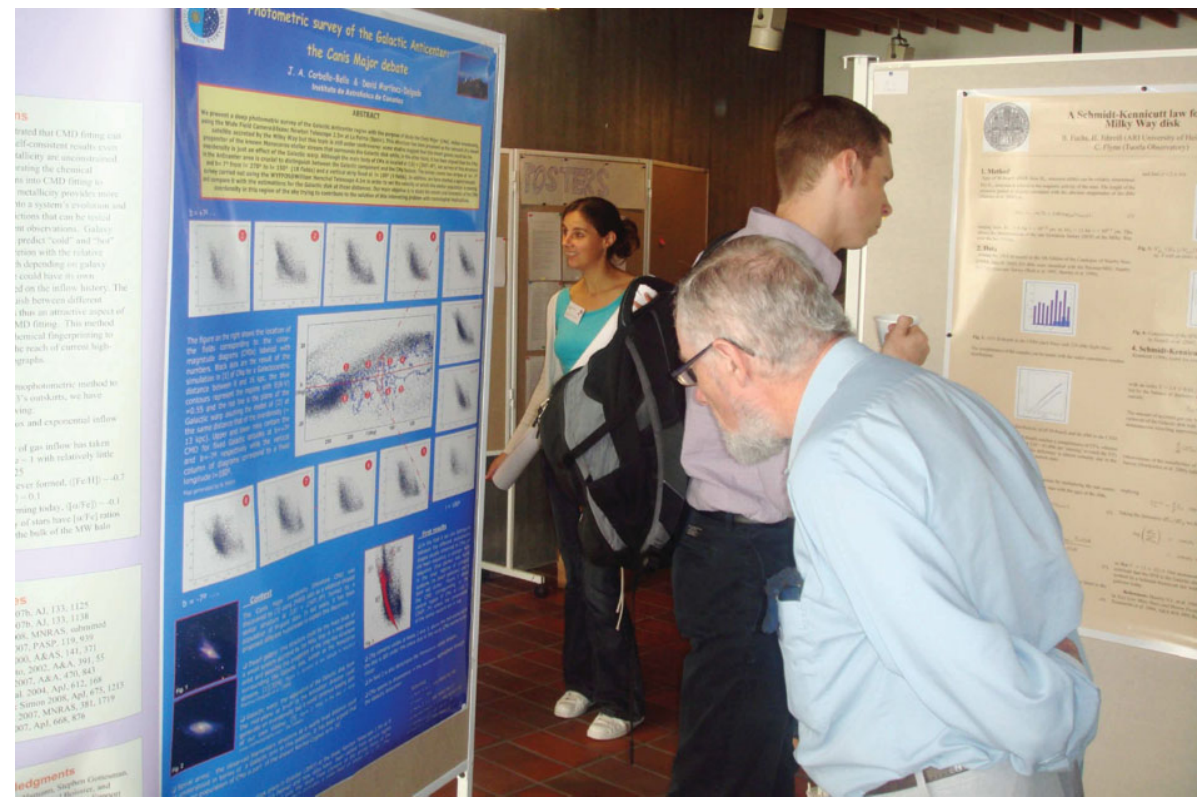

Posters were studied eagerly as well, here by Tom Lloyd Evans. 